\title{
Theoretical Investigation of the Interaction Between a Compressor and the Components During Surge
}

\author{
Jürgen Brumm, Hassan Kalac and Ingolf Teipel \\ Institute for Mechanics, University of Hannover, Germany
}

\begin{abstract}
Problems of compressor instability have been of concern not only to aircraft engine designers but also for example to operators of industrial power plants. Two types of flow instabilities are known and in this paper only one of them, the surge phenomenon, will be discussed in detail. For the occurrence of these heavy pressure fluctuations the volume of the neighbouring plenum chamber is the important quantity.

Here the basic compressor system consisting of a compressor, a duct and a plenum will be analysed in order to show the complete unsteady pressure field. The theoretical model is based on the application of mass, momentum and energy conservation equations of one-dimensional flow. The set of differential equations of hyperbolic type is solved numerically by a 'predictorcorrector' scheme. The method itself is an explicit procedure first given by MacCormack $19 /$. At the interfaces between two components the coupling conditions for pressure and flow velocity are taken from the method of characteristics. The behaviour of the compressor is determined by its performance map, which describes the relationship between pressure and mass flux for all conditions from stable performance to inverse mass flow. For the dynamic change of the pressure development a special compressor characteristic taking into account the unsteady effects is used. This model function is chosen according to a time lagging element often employed in control theory.

To demonstrate the capability of the described method variations of different parameters such as mass flow rate, volume behind the compressor, etc. will be presented. The pressure-time history will be compared with experimental data published in the literature. Also the calculated surge frequencies will be discussed. Furthermore it will be examined how a working point of the compressor moves from a stable part of the characteristic to another one, stable or unstable. Finally the general time dependent surge performance is considered in comparison to the quasi-steady characteristic.
\end{abstract}

\section{Nomenclature}

a

A

velocity of sound $[\mathrm{m} / \mathrm{s}]$

cross section of the compressor (averaged) $\left[\mathrm{m}^{2}\right]$

e specific energy $[\mathrm{J} / \mathrm{kg}]$

$\mathrm{F}$ vector, defined in eq. (7)

$G$ vector, defined in eq. (7)

$\mathrm{h}$ specific enthalpy $[\mathrm{J} / \mathrm{kg}]$

$\mathrm{K}_{\mathrm{R}}$ force (friction) [N]

$\dot{\mathrm{m}}$ mass flow rate $[\mathrm{kg} / \mathrm{s}]$

$\mathrm{p}$ pressure $\left[\mathrm{N} / \mathrm{m}^{2}\right]$

$\mathrm{q} \quad$ specific heat production $[\mathrm{J} / \mathrm{kg}]$ s specific entropy $[\mathrm{J} / \mathrm{kgK}]$

$t$ time [s]

$\mathrm{T}_{1}$ time constant [s]

$\mathrm{U}$ vector, defined in eq. (7)

$V$ volume of the compressor $\left[\mathrm{m}^{3}\right]$

$\dot{V}$ volume flow rate $\left[\mathrm{m}^{3} / \mathrm{s}\right]$

w velocity $[\mathrm{m} / \mathrm{s}]$

$W_{\mathrm{t}}$ specific work $[\mathrm{J} / \mathrm{kg}]$

$\mathrm{x}$ axial coordinate [m]

$\pi \quad$ pressure ratio

$\pi$ dyn pressure ratio (dynamic)

$\pi_{\text {ST }}$ pressure ratio (quasi static)

$\rho$ density $\left[\mathrm{kg} / \mathrm{m}^{3}\right]$ 


\section{Introduction}

In today's compressor units of high pressure loading the performance map shows that the distance between the design point and the limit of flow stability is not very large. Small changes of the mass flow rate of the whole system may therefore lead to unsteady phenomena. Two types of oscillations are known: rotating stall and surge.

Rotating stall occurs if the flow rate through the machine is throttled gradually and if as a consequence the angle of attack at the blades of different stages differs from the optimal position. One or more cells of retarded flow appear and they usually rotate in the same direction as the impeller but with a speed less than that of the rotor. The time averaged mass flow remains nearly constant. Because of the low flow rate essential overtem. peratures may appear in the burner of the turbine. Furthermore it is very difficult to return to an unstalled situation.

Surge, on the other hand, means that during the unstable process the mass flow oscillates with large amplitude. The frequency of surge is approximately of an order lower than the value of rotating stall. The complete flow field in the compressor is affected by this mechanism. It is not very difficult to return to a stable operation by opening again the throttle.

Even today the onset of these instabilities cannot be predicted correctly by simple methods. One has to deal with nonlinear effects and therefore complicated procedures for the determination of the essential features have to be used. In former times the beginning of instability was often associated with a throttle line to be tangent on a compressor characteristic. This criterion is often too simple to describe the phenomena that can be observed. Only for the static instability one can apply this condition. For the dynamic stability additional information concerning the interaction of all the waves in the whole system has to be included. The occurrence of surge cycles can be expected if the energy fed into the oscillations by the compressor is equal to the work done by the mechanical system together with the damping effects. Because of the amplitude and the low frequency of these oscillations the compressor may start from a point of performance map completely free from stall and passes values with reversed flow.

In this paper a theoretical nonlinear model of the unsteady flow field in the whole compression system will be developed. Particular emphasis is directed towards the history of the pressure development in the compressor. Numerical results will be shown and compared with experimental data for a surge cycle.

\section{The Fluid Dynamic Model}

The compression system (Fig. 1) which will be investigated in this paper consists of the inlet with a suction tube, the compressor unit, a pipe to the plenum chamber and a throttle with the exhaust pipe. Air enters the suction pipe and passes through the compressor. The mass flow rate will be controlled by a throttle valve.

To illustrate the different instabilities, a pressure recording from $/ 1 /$ is shown in Fig. 2. The operating point of the compressor has been moved to a smaller flow rate than under design conditions. In the first part of the graph a more or less quasisteady pressure distribution can be seen and then

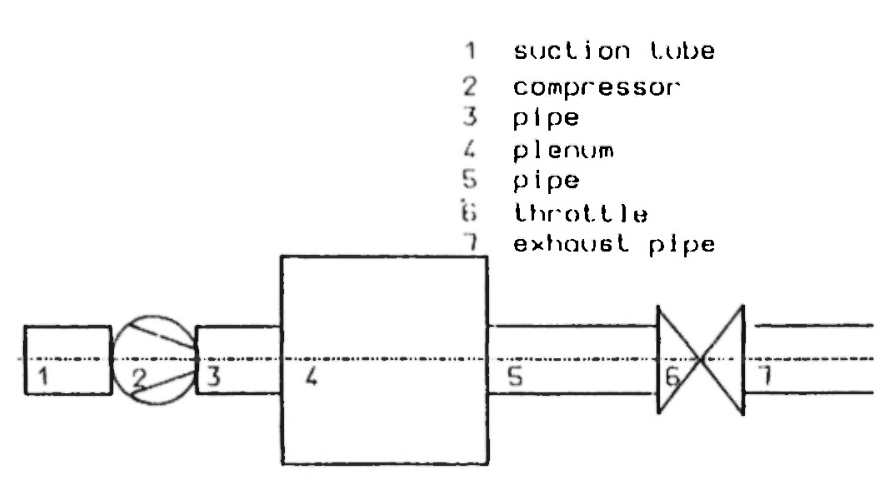

Fig. 1. Basic elements of a piping system.

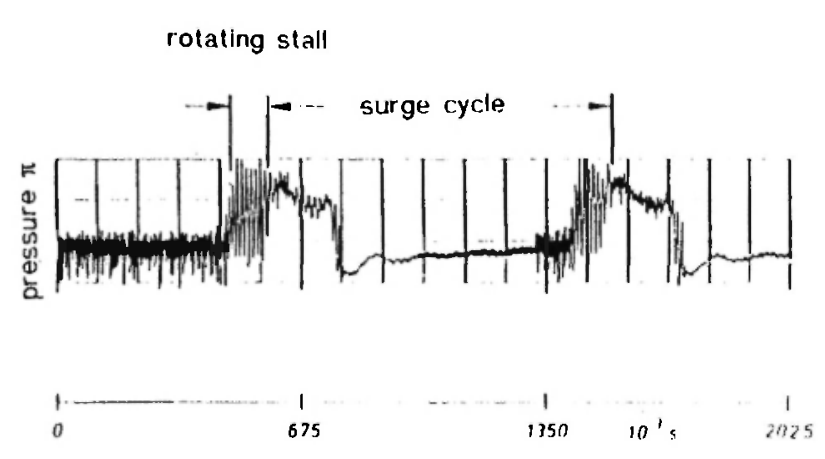

Fig. 2. Experimental results of unstable system behaviour. 
a transition to a flow situation with rotating stall properties happens. Finally a deep surge cycle occurs. The change from rotating stall to surge depends not only on the characteristic of the compressor itself, but also on the characteristic of the throttle and the plenum chamber. A large reservoir can accumulate the pressure like a mechanical spring. The potential energy makes it possible that one can observe even back flow at a surge cycle.

To calculate the dynamic behaviour of the com. pression system two different approaches are known in the literature:

a) Lumped Parameter Analysis and

b) Discretized Flow Analysis.

In the first method all parts of the system have been represented by simple mass-spring combinations sometimes including also damping effects. So even with a complicated geometry one obtains finally an ordinary differential equation for the determination of the mass flow rate. This approach has been used by several authors $/ 2,3 /$.

The second procedure starts from the basic fluid dynamic equations. This system of nonlinear partial differential equations has to be solved numerically. Results using this method have been given in $/ 4,5$, $10 /$. In the analysis the compressor is represented by its characteristic obtained by measurements. For the dynamic behaviour of the whole compression system Ohashi /6/ suggested to introduce a formulation for the pressure development by applying an equation with time lagging terms. So it is possible to describe more realistic the history of pressure rise or drop. A similar characterisation of the flow behaviour in the compressor will be used in this paper.

\section{The Mathematical Analysis}

In order to calculate the flow through the whole system including pressure waves with different kinds of reflection conditions the conservation equations for mass, momentum and energy will be applied. For one-dimensional unsteady flows of a compressible fluid the following equations will be given:

continuity equation:

$$
\frac{\partial \rho}{\partial \mathrm{t}}+\frac{\partial \rho \mathrm{w}}{\partial \mathrm{x}}=0
$$

momentum equation:

$$
\frac{\partial \rho \mathrm{w}}{\partial \mathrm{t}}+\frac{\partial\left(\rho \mathrm{w}^{2}+\mathrm{p}\right)}{\partial \mathrm{x}}=\mathrm{K}_{\mathrm{r}}
$$

energy equation:

$$
\frac{\partial \rho\left(\mathrm{e}+\frac{w^{2}}{2}\right)}{\partial \mathrm{t}}+\frac{\partial \rho \mathrm{w}\left(\mathrm{h}+\frac{\mathrm{w}^{2}}{2}\right)}{\partial \mathrm{x}}=q+\mathrm{w}_{\mathrm{t}}
$$

Here in the momentum equation viscous effects have been introduced in an approximate manner by $K_{R}$. In the energy equation specific terms $q$ and $W_{t}$ for the added heat and the mechanical work have been included. The equations are written in the conservative form, because also the existing discontinuities like shock waves shall be represented in the numerical computation. The compressor itself will be described by the characteristics of the performance map together with an equation defining the pressure history. The basic information for such a formulation can be obtained from measurements. Although in most cases these compressor characteristics for constant number of revolutions are discontinuous at the first stability limit, a continuous polynomial expression has been used in this paper for the mathematical description. For the computation a third order polynomial for $p=$ $\mathrm{f}(\mathrm{m})$ beginning from a stable operating point until the zero-mass flux, is introduced and for the region with reversed flow a different third order polynomial is established. Of course the only use of the steady state characteristics postulates that there is an immediate response between the change of mass flow and the pressure rise. This description leads to an error which grows with increasing frequency of the oscillation of the mass flow rate. In order to demonstrate the different reactions of the system, the pressure development for high and low frequencies has been shown in Fig. 3 and Fig. 4. The dotted line represents the steady compressor characteristic. For the low-frequency case (Fig. 3) the deviation from the quasi-steady characteristic is very small such that it is satisfactory to keep the steady characteristic for all computations. The error can be neglected. But if the frequency of the oscillations of the flow rate increases (Fig. 4), the instantaneous operating point does not coincide any longer with the steady charac- 


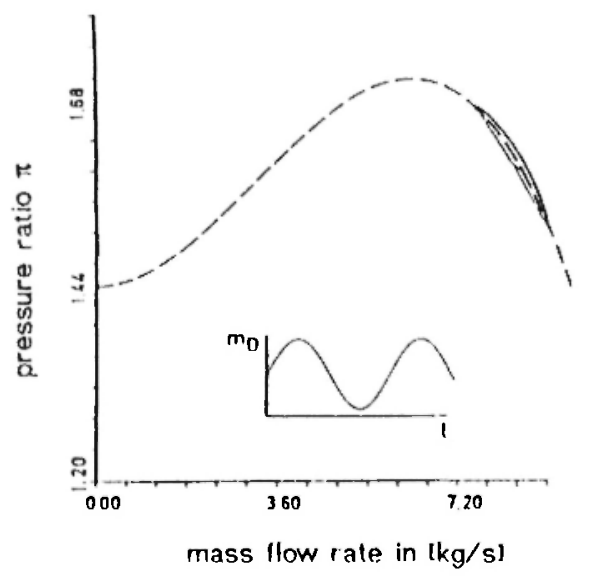

Fig. 3. Dynamic response (low frequency).

teristic. In this case it is necessary, even in a stable situation from the static point of view to try an unsteady formulation of a compressor characteristic if one is interested in details of the flow field.

Investigations of this phenomenon have shown that such a problem can be described by an approach often used in control theory. The proportional action controller of first order has similar properties which appear here in the pressure-mass flow response. Therefore it seems obvious to transfer the same expression to the mathematical model used in this report. One obtains

$$
\mathrm{T}_{1} \frac{\mathrm{d}}{\mathrm{dt}}\left[\pi_{\mathrm{dyn}}(\mathrm{t})\right]+\pi_{\mathrm{dyn}}(\mathrm{t})=\pi_{\mathrm{ST}}(\mathrm{t})
$$

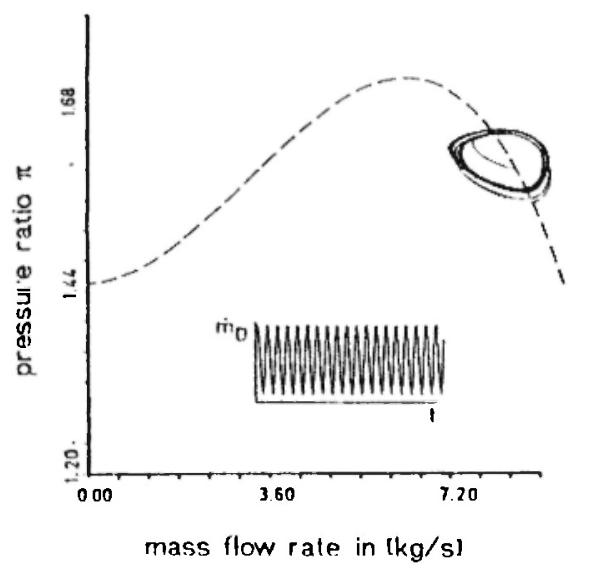

Fig. 4. Dynamic response (high frequency).
$\pi_{\mathrm{ST}}$ is the pressure ratio from the quasi-steady characteristic and $\pi_{\mathrm{dyn}}$ the new defined dynamic pressure ratio. $\pi_{d y n}$ is the most significant value for the computation in the system. The time constant $T_{1}$ is a quantity which can be calculated by the volume of the compressor, the average crosssection $\mathrm{A}$ and the mean value of the velocity:

$$
\mathrm{T}_{1}=\frac{\mathrm{V}}{\mathrm{AW}}
$$

It would be very important to have experimental data which can determine this parameter. From the physical point of view one can explain $T_{1}$ as the average time that a particle needs to pass through the compressor.

\section{The Numerical Solution}

For the calculation of the unsteady flow field with its quantities pressure, velocity and density the continuing equation, the momentum equation and the energy equation have to be solved. Here also the variable cross-section $\mathrm{A}$ will be introduced.

$$
\frac{\partial}{\partial \mathrm{t}}\left[\begin{array}{l}
\rho \mathrm{A} \\
\rho \mathrm{wA} \\
\rho \mathrm{A}\left(\mathrm{e}+\frac{\ddot{*}^{2}}{2}\right)
\end{array}\right]+\left[\begin{array}{ll}
\rho \mathrm{wA} \\
\frac{\hat{\sigma}}{\partial \mathrm{x}} & \mathrm{A}\left(\rho \mathrm{w}^{2}+\mathrm{p}\right) \\
& \rho \mathrm{wA}\left(\mathrm{h}+\frac{\mathrm{w}^{2}}{2}\right)
\end{array}\right]
$$

$$
+\left[\begin{array}{ll}
0 & \\
--K_{\mathrm{r}} & \\
-q-w_{t}
\end{array}\right]=0
$$

$\mathrm{K}_{\mathrm{R}}$ represents again a global expression for the viscous force, and $W_{t}$ can be calculated from the losses of a polytropic process. These three nonlinear partial differential equations are of hyperbolic type. Therefore one has to take into account the existence of nonlinear continuities, e.g. shock waves. As an analytic solution cannot be found, a numerical method has been applied. Starting from eq. (6) in matrix formulation

$$
\frac{\partial U}{\partial t}+\frac{\partial F}{\partial x}+G=0
$$


a two-step procedure first given by MacCormack /9/ yields

predictor step

$$
\widetilde{U}_{j}^{n+1}=U_{j}^{n}-\frac{\Delta_{t}}{\Delta x}\left[F_{j+1}^{n}-F_{j}^{n}\right]-\Delta t G_{j}^{n}
$$

corrector step

$$
\begin{aligned}
& U_{j}^{n+1}=U_{j}^{n}-\frac{1}{2} \frac{\Delta t}{\Delta x}\left[F_{j+1}^{n}-F_{j}^{n}+\right. \\
& \left.+\tilde{F}_{j}^{n+1}-\widetilde{F}_{j-1}^{n+1}\right]-\frac{1}{2} \Delta t \widetilde{G}_{j}^{n+1}
\end{aligned}
$$

It is an explicit scheme and the accuracy is of second order. For this scheme the Courant-FriedrichLewy condition has to be used in order to calculate the step size which guarantees numerical stability.

The initial values are extrapolated from the steady-state solution. And finally the boundary conditions for the entrance and the exit of the tube have to be formulated dependant on the flow direction.

In order to connect the different parts of the piping system during the computation, for example the suction pipe and the compressor, the method of characteristics has been used. As it was already mentioned one has to add further conditions for the flow through the compressor, e.g. the characteristics of the performance map and eq. (4) describing the pressure history.

For the start of the whole calculation the crosssection of the throttle behind the plenum chamber has been changed. So pressure waves will run through the compression system and will be reflected several times at different points. For the numerical procedure the compression system is divided in 100 cells of equal length.

\section{Results of the Calculation}

Calculations of three different cases have been carried out:

First the transient behaviour of the compressor has been studied when the study operation point will move to another stable position.

Second the variation of an operation point near the stability limit will be discussed.
Third the surge phenomenon including the complete cycle will be calculated by regarding all nonlinear wave interaction effects.

In the first case the influence of periodic changes of the mass flow rate on the dynamic pressure variations has been discussed. It could be shown as can be seen from Fig. 2 - that the deviation of the operation point from the steady compressor characteristic is small. Finally another operation point in the stable region will be reached. One can use the steady compressor characteristic for all calculations. The behaviour near the maximum of the performance curve is studied in the second case. It depends on the frequency and the amplitude of the change of mass flow rate if one ends up in an unstable situation. Nevertheless the dynamic part of the compressor characteristic becomes important as can be seen in Fig. 3. It is a very sensitive calculation and it can be expected that for characteristics with positive slope one needs the pressure-history evaluation.

The third problem will be described in more details furtheron. From the last case above one has learnt that the dynamic variation of the pressure has to be introduced if the question whether the flow is stable or not is raised.

A first result of this computation is shown in Fig. 5. The difference between the steady and the dynamic part of the pressure is plotted versus time. The value of the time constant $T_{1}$ in eq. (4) is here $0.08 \mathrm{~s}$. It shall be mentioned that the zero-pressure line does not coincide with the time-axis. Over the whole time interval in Fig. 5 there is a significant deviation from the steady pressure distribution. Of course a certain periodicity and a strong nonlinear interaction can be detected. An estima-

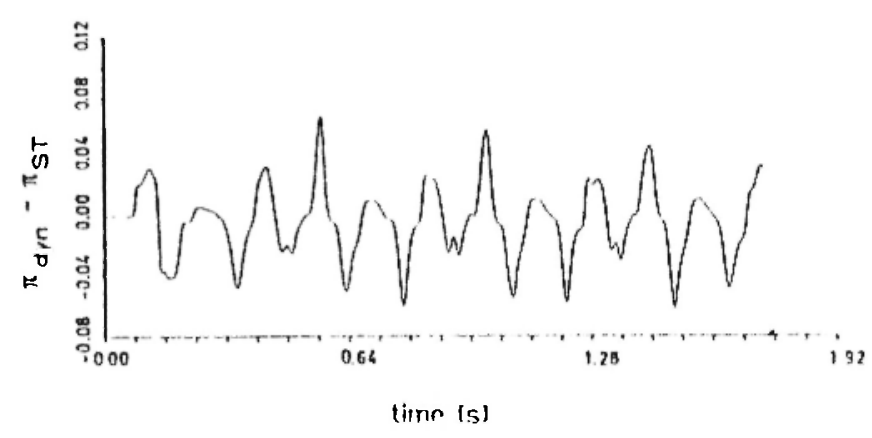

Fig. 5. Difference between dynamic and quasi-static characteristic with $T_{1}=0.08 \mathrm{~s}$. 


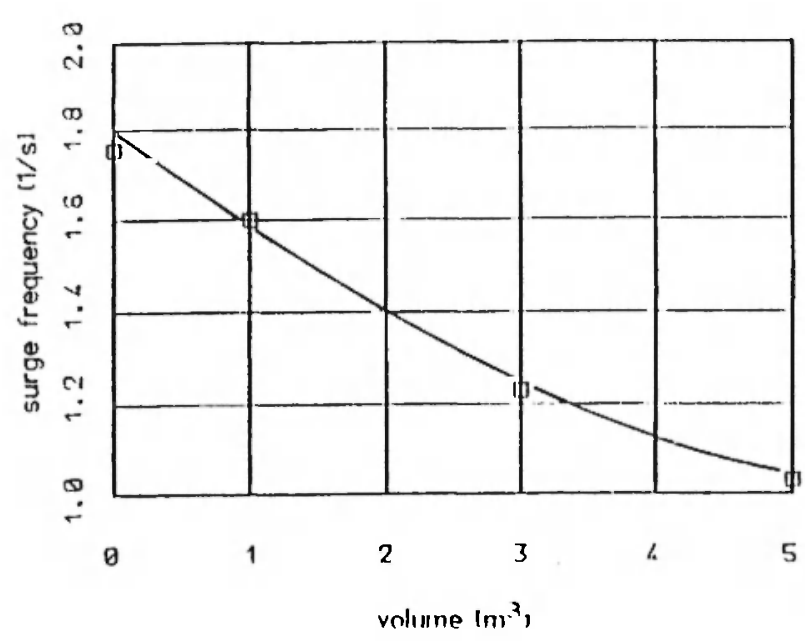

Fig. 6. The effect of volume on surge frequency.

tion yields that the pressure disturbance is approximately $8 \%$.

From the whole calculation one gets some information about the frequency of a surge cycle. In the next figure (Fig. 6) the surge frequency is plotted versus the volume of the plenum chamber. This volume means an additional part to the volumes contained in the pipes. The frequency decreases with increasing volume. This can be explained by the following consideration: The time for filling up the plenum chamber becomes larger and therefore the pressure growth needs more time.

In Fig. 7 the surge frequency is shown as a function of the time constant $T_{1}$. The frequency decreases again because the pressure rise is now slowed down. For comparison reasons measurements have been added in this figure. In the experiments the scattering of the data obtained is very large. So they can be found in the shadowed band and vary from 1.6 to $1.91 / \mathrm{s}$. If one looks back to the value of $T_{1}$ in the calculation above, a constant of $0.08 \mathrm{~s}$ seems to be realistic for the experimental and practical operations.

The next two figures show the surge cycle with its characteristics plotted in the performance map. The region with the dots visualizes the time behaviour. Starting from point 1 under stable conditions (Fig. 8), the associated dynamic pressure line, the dashed line, deviates from the steady characteristics $\pi_{\mathrm{ST}}$ via point 2,3 and 4 until the region of backflow (point 5). There is only a small pressure drop. Until point 8 with the pressure minimum, the pressure variation follows more or less the pre-

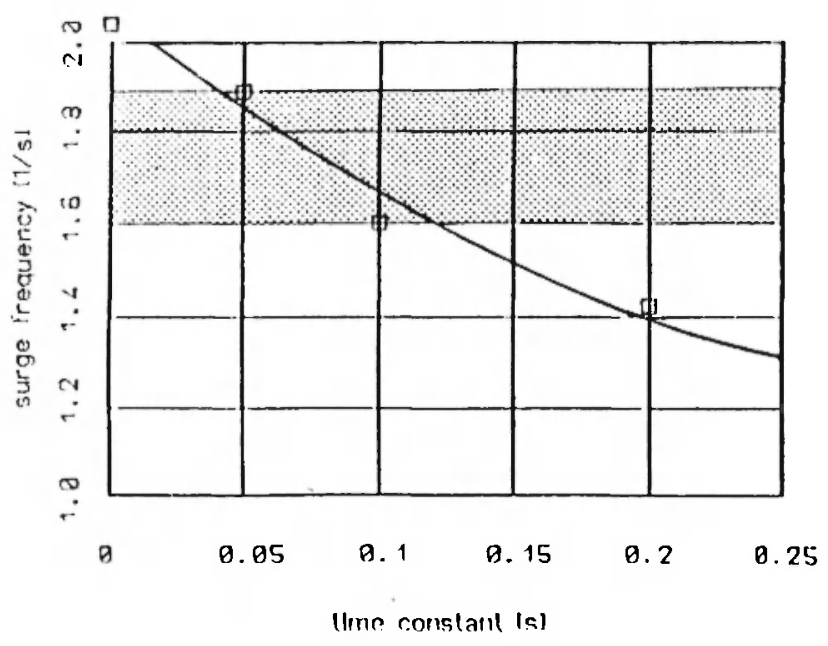

Fig. 7. The effect of time constant on surge frequency.

scribed compressor characteristic. Finally, the mass flow rate increases again and comes back to point 1 .

A similar process has been studied experimentally by Dibelius and Rauhut /4/ and these results have been shown in Fig. 9. Despite of the different values of the abszissa and if one keeps in mind how difficult these measurements are to be carried out, the principal agreement between Fig. 8 and Fig. 9 is satisfactory. It should be mentioned that point 1 is not on the characteristic, because the measurements of the surge cycles are not started from the stable point, but represent a surging machine.

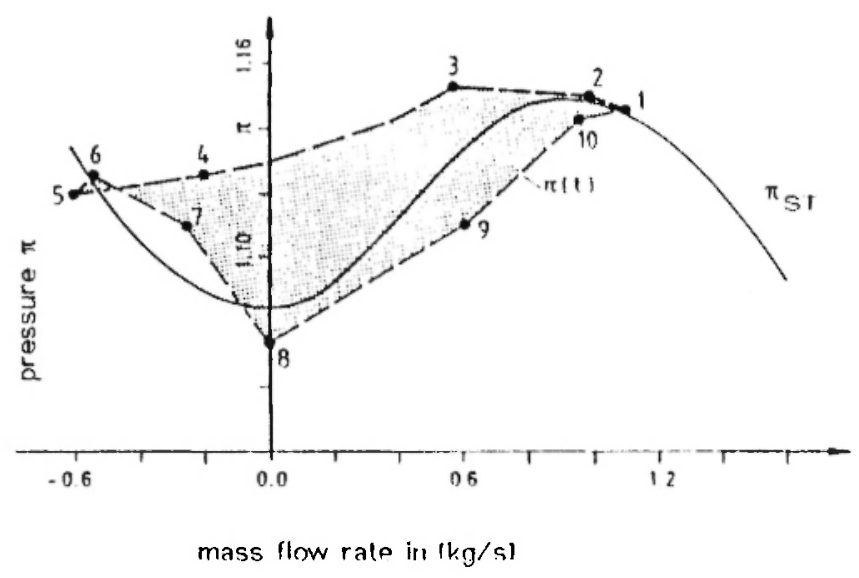

Fig. 8. The computation of a surge cycle in the performance map. 


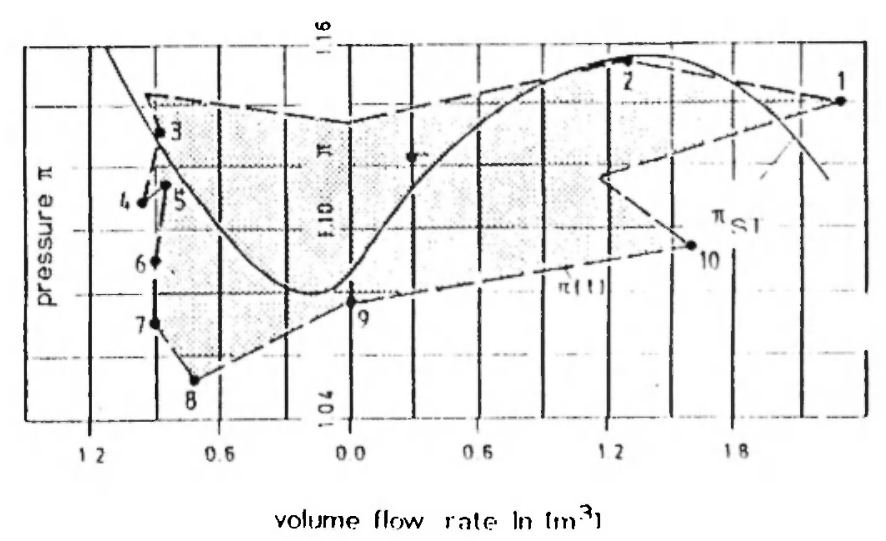

Fig. 9. The measurement of a surge cycle in the performance map.

\section{Concluding Remarks}

The nonlinear model developed in this paper describes obviously the important features of the different transient processes in a compression system adequately. So the surge frequency can be obtained in satisfactory agreement with experiments. The basic assumption is the introduction of an equation for the pressure-history. Here a time constant $T_{1}$ represents the delay of the interaction between pressure and mass flow variations. Unfortunately one cannot find experimental data for $T_{1}$. So one has to estimate reasonable values. But indirectly one can get information for $T_{1}$ through a parameter simulation of the volume of the plenum.

Finally one may conclude from this study that the main point of further investigation has to be directed towards the determination of the unsteady compressor characteristic. So one has to discuss separation phenomena with respect to the influence of performance map. Also the geometry of the cascade in the different stages has to be included in the theoretical model.

\section{Acknowledgements}

The authors would like to thank the "Deutsche
Forschungsgemeinschaft" in Germany for the support of this project. The computation were made on the CYBER 990 of the RRZN (Regionales Rechenzentrum Niedersachsen).

\section{Discussion of $/ 10 /$}

In the beginning of 1988 we have been noticed by the reviewers of our paper that a similar subject has been studied by DAVIS and O'BRIEN /10/.

\section{References}

1. U. Schlamann, I. Teipel and W. Riess, "Experimentelle Untersuchung der Strömungsphenomene des Rotating Stall und des Pumpens bei mehrstufigen, hochbelasteten Axialverdichtern". VDI-Fortschrittsberichte, Reihe 7, Nr. 91 (1985).

2. E.M. Greitzer, "The Stability of Pumping Systems - The 1980 Freeman Scholar Lecture". ASME Journal of Fluids Engineering, 103, 193-242, June (1981).

3. Th. Carolus and K.O. Felsch, "Der Einfluss des dynamischen Verhaltens der Geblasekennlinie auf Pumpgrenze und Pumpstarke einer lufttechnischen Anlage". VDl-Berichte Nr. 487 (1983).

4. G. Dibelius and H. Rauhut, "Vorausberechnung der Pumpgrenze und des Pumpverhaltens einer Verdichteranlage". VDI-Berichte Nr. 487 (1983).

5. H. Kalac, "Beitrag zur rechnerischen Simulation des instationären Betriebsverhaltens von Verdichteranlagen". VDI-Fortschrittsberichte, Reihe 7, Nr. 118 (1987).

6. H. Ohashi, "Analytical and Experimental Study of Dynamic Characteristics of Turbopumps". NASA Technical Note D-4298 (1968).

7. E.M. Greitzer and F.K. Moore, "Stalled Flow Performance for Axial Compressors", Part II, ASMEPaper 85-GT-172.

8. E.M. Greitzer and S.G. Koff, "Stalled Flow Performance for Axial Compressors", Part I, ASME-Paper 84-GT93.

9. R.W. MacCormack, "A Numerical Method for Solving Equations of Compressible Viscous Flow". AIAAPaper 81-0110 (1981).

10. M.W. Davis and W.F. O'Brien, "A Stage-by-Stage PostStall Compression System Modeling Technique." AIAA-Paper 87-2088 (1987). 
Brought to you by | University of Arizona Authenticated 